\title{
La valuación de empresas cotizadas en México, mediante la metodología del modelo de Flujo de Efectivo Disponible Relaciones con la valuación del mercado
}

\author{
María Luisa Saavedra*
}

\begin{abstract}
Resumen
El objetivo de esta investigación fue aplicar el modelo de Flujo de efectivo disponible para determinar el valor de un grupo de empresas que cotizan en la Bolsa Mexicana de Valores para comparar los valores obtenidos con el precio de mercado.

La revisión de la literatura del modelo de Flujo de Efectivo Disponible permitió identificar y describir las principales variables que contiene el mismo, con el fin de proponer una metodología de aplicación a las empresas que cotizan en la Bolsa Mexicana de valores, considerando que sólo se contaba con información pública. Con la metodología propuesta se aplicó el modelo de Flujo de Efectivo Disponible a las empresas que cotizan en la Bolsa Mexicana de Valores, con el fin de determinar su valor y tratar de encontrar si existe algún patrón de comportamiento del modelo al valuar a este conjunto de empresas, por un periodo de 10 años de 1991 a 2000.

Encontramos que el modelo de Flujo de Efectivo Disponible sigue una tendencia muy similar al precio de mercado, lo cual estaría indicando a este modelo como el más adecuado para valuar a este conjunto de empresas tomando, desde luego, el valor de mercado como patrón de referencia.

Palabras clave: Flujo de efectivo disponible, valuación de empresas, costo de capital, Bolsa Mexicana de Valores, Precio de Mercado.
\end{abstract}

Fecha de recepción: 17/04/2006

Fecha de aceptación: 11/05/2007

\footnotetext{
* Profesora investigadora de la Universidad Autónoma del Estado de Hidalgo. Correo electrónico: maluisasaavedra@yahoo.com
} 
María Luisa Saavedra

\title{
Valuation of Companies Quoted in Mexico, by means of the Methodology of the Model of Cash flow Available. Relations with Valuation of the Market
}

\begin{abstract}
The objective of this investigation was to apply the model of Cash flow available to determine the value of a group of companies that quote in stock-market Mexican of Values, and of comparing the values obtained with the price of market.

The revision of the Literature of the model of Cash flow Available allowed to identify and to describe the main variables that he himself contains, with the objetive of proposing a methodology of application to the companies that quote in stock-market Mexican of values, considering that single was counted on public information. With the propose methodology the model of Cash flow Available was applied to the companies that quote in stock-market Mexican of Values, with the purpose of determine their value and of try to find if some pattern of behavior of the model when estimating to this set of companies exists, by a period of 10 years from 1991 to 2000.
\end{abstract}

We found that the model of Cash flow Available follows a very similar tendency at the cost of market, which would be indicating to this model like the most adapted to estimate to this set of companies taking, of course, the value from market like reference pattern.

Key words: Cash flow available, Valuation of companies, Capital cost, Mexican stock market of values, Price of market.

\section{Introducción}

$\mathrm{C}^{1}$ modelo de Flujo de Efectivo Disponible ${ }^{1}$ es uno de los principales modelos de ¿valuación de empresas en el medio financiero. Este modelo determina el valor de la empresa por la capacidad de generación de flujos de efectivo y no por sus utilidades. En este sentido, Carvallo (1995: 34) señala que el valor de la empresa aplicando este modelo depende de la capacidad de la misma de generar efectivo, el cual se puede conocer determinando los flujos de efectivo operativos.

${ }^{1}$ Entre quienes han desarrollado el modelo FED aplicado a la valuación de empresas tenemos: Copeland et al. (1994), Carvallo (1995), Franks (1985), Damodaran (1994), Mascareñas (2000). 
Por esta razón este modelo centra su atención en el cálculo y análisis de los "generadores de valor", 2 que son los que crean valor dentro de la empresa y por lo tanto generan los flujos de efectivo necesarios para la operación de la misma. ${ }^{3}$

El objetivo de esta investigación es identificar y describir las variables que contiene el modelo de valuación de empresas de Flujo de Efectivo Disponible y proponer una metodología de aplicación del mismo en las empresas que cotizan en la Bolsa Mexicana de Valores. Todo con el fin de determinar de qué forma valúa este modelo a las empresas mencionadas.

Este modelo es uno de los más conocidos y utilizados en el medio financiero, sin embargo aún no se le ha dado la difusión debida por lo cual su aplicación es limitada. De esta manera entonces lo que se pretende en esta investigación es demostrar que es viable su aplicación en las empresas que cotizan en la Bolsa Mexicana de Valores y que los resultados de esta aplicación son congruentes con la teoría financiera y con las pruebas empíricas que han hecho otros investigadores.

La pregunta de investigación es: ¿Cuáles son las variables que contiene el modelo de valuación de empresas de Flujo de Efectivo Disponible y cómo se puede aplicar este modelo a las empresas que cotizan en la Bolsa Mexicana de Valores? y, ¿de qué manera el modelo ${ }^{4}$ de valuación ${ }^{5}$ de empresas de Flujo de Efectivo Disponible, valúa a las empresas que cotizan en la Bolsa Mexicana de Valores?

\footnotetext{
${ }^{2}$ Según Copeland et al. (1994: 33), estos pueden ser el crecimiento en ventas, el margen operativo, la inversión adicional en capital de trabajo, la inversión adicional en activos fijos, entre otros.

${ }^{3}$ Entre quienes han desarrollado el modelo FED aplicado a la valuación de empresas tenemos: Copeland et al. (1994), Carvallo (1995), Franks (1985), Damodaran (1994), Mascareñas (2000).

${ }^{4}$ Según Thierauf (1987: 32): "El modelo es una representación o abstracción de una situación u objeto reales, que muestra las relaciones (directas e indirectas) y las interrelaciones de la acción y la reacción en términos de causa y efecto... Una de las razones básicas para el desarrollo de modelos es la de descubrir cuáles son las variables importantes o pertinentes. Este descubrimiento está estrechamente asociado con la investigación de las relaciones que hay entre las variables".

${ }^{5}$ En el contexto de la presente investigación, no se considera la teoría del valor, sólo el valor muy precisado por una minimización financiera, es decir el valor se reduce a términos financieros. En este sentido, Van Horne (1993: 102) señala: "El valor para la empresa se crea mediante las inversiones de capital que aprovechan las oportunidades de rendimientos excedentes, aquéllas que proporcionan rendimientos en exceso con respecto a los que los mercados financieros requieren para el riesgo supuesto". Por otra parte, Rappaport (1986: 81) indica que las vías para crear valor son el atractivo de la industria y la ventaja competitiva. Por último, Taggart (1994: 69), señala que los determinantes financieros de la creación de valor son: el rendimiento sobre el capital, la tasa de crecimiento del capital y el costo de capital.
} 
María Luisa Saavedra

\section{Modelo de Flujo de Efectivo Disponible}

El modelo de Flujo de Efectivo Disponible (FED), ${ }^{6}$ de acuerdo a lo propuesto por Copeland et al. (1994: 132), indica que el valor del capital propio de una empresa es igual al valor presente de los flujos de efectivo disponibles, menos el valor presente de los flujos pagados a todos los acreedores (éstos, disponibles a la tasa de costo de capital de la empresa). Asimismo, Copeland et al. (1994: 133), considera sólo el flujo de efectivo operativo señalando una metodología para su cálculo; de igual forma considera sólo la deuda con costo. Además sugiere calcular los flujos de efectivo para un periodo explícito de siete años y un periodo de pronóstico del valor continuo bajo el supuesto de empresa en marcha.

Es necesario señalar que este modelo determina el valor de la empresa por su capacidad de generación de flujos de efectivo y no por sus utilidades (Carballo, 1995: 34). Así también, Franks (1985: 352) coincide al señalar que el valor de la empresa se puede obtener sustrayendo el valor presente de las deudas a largo plazo del flujo de efectivo de la misma. Al respecto, Damodaran (1994: 122) dice que el hecho de considerar el apalancamiento lo hace muy útil cuando el de la empresa es significativo. Mascareñas (2000: 340), por otra parte, indica que se puede llegar a este mismo valor obteniendo el valor de las acciones, descontando los flujos de caja disponibles para los accionistas al costo de capital de las acciones comunes y sumándole el valor de la deuda. También es importante el señalamiento que hace Copeland et al. (2000: 137), en el sentido de que hay dos directrices claves del flujo de efectivo y en última instancia del valor: la tasa de crecimiento de los ingresos, utilidades y capital de la empresa; y el rendimiento sobre el capital invertido.

Muchos autores le han hecho adaptaciones al modelo a fin de aplicarlo a situaciones específicas. Así, Desmet (2000: 149) sugiere usar el modelo FED combinado con un análisis microeconómico y escenarios de probabilidades para valuar compañías de alto crecimiento, como lo son las empresas de Internet. Nygard (1999: 68), a su vez, señala que se debe aplicar un escenario de probabilidades y hallar con este modelo el valor de la empresa, no como un punto fijo, sino como un rango de valor. Por otra parte, Luehrman (1997: 146) recomienda el uso del modelo de Valor presente ajustado (APV), en el cual se calculan los flujos de efectivo disponibles y luego se le suman los efectos del financiamiento, para reflejar en el valor de la empresa los beneficios fiscales por deducción de intereses.

${ }^{6}$ También se le conoce como FCF, por sus siglas en inglés (Free Cash Flow). 
Varios investigadores han intentado comprobar la validez del modelo de FED. De esta manera, Christofi (1999: 38) señala que la validez del mismo se encuentra en el supuesto, de que el valor de la empresa se puede reflejar en sus oportunidades de crecimiento de largo plazo, lo que se refuerza con los hallazgos de Rappaport (1986: 31), el cual encontró que el $60 \%$ del valor de mercado de la empresa es atribuible a las ganancias que ocurren en un horizonte inmediato de 5 años. Basado en estos supuestos, Christofi (1999: 38) realiza una investigación en la que, con base en el valor de las acciones de la empresa, el flujo de efectivo anual y el costo del capital propio determinan la tasa de crecimiento de largo plazo de los flujos de efectivo de la empresa, llegando a una conclusión positiva de la validez de este modelo.

Este modelo también tiene sus debilidades; en este sentido, Nelson (2000: 538) señala que en el flujo de efectivo disponible la tasa de descuento refleja el riesgo de la empresa. De este modo los expertos eligen una tasa de descuento que corresponde al costo de capital de la empresa, y no la tasa de retorno que requieren los inversionistas potenciales de la empresa, induciendo así a errores de estimación.

El modelo FED es uno de los más conocidos y utilizados en el medio mexicano; se puede aplicar para determinar un valor exacto como en el modelo original propuesto por Copeland et al. (1994: 132); o también en las adaptaciones sugeridas por Desmet (2000: 149), Nygard (1999: 68) o Luherman (1997: 146), para determinar diversos valores con sus respectivos escenarios o un rango de valor que eleve la probabilidad de éxito en la valuación, así como la incorporación del efecto del financiamiento.

El considerar variables como el flujo de efectivo, el costo de capital, el valor continuo, el flujo de la deuda y la tasa de crecimiento para proyectar los flujos de efectivo, entre las principales, hace que este modelo sea más completo y más congruente en el cálculo del valor de la empresa, ${ }^{7}$ ya que considera la misma como una inversión que tiene que generar un flujo continuo de dinero líquido, para que la empresa esté en condiciones de seguir creciendo y financiando con su propia operación, su crecimiento y expansión en un mercado competitivo.

La esencia de este modelo es que el valor del capital propio de una empresa (valor intrínseco), es igual al valor presente de los flujos de efectivo disponibles, menos el valor presente de los flujos pagados a todos los acreedores (deuda con costo) de la empresa (Copeland, et al. 1994: 132), ${ }^{8}$ (véase tabla 1).

\footnotetext{
${ }^{7}$ Una comparación del modelo FED, con otros usados en el medio financiero, puede consultarse en Opiela (2000) y Penman (1998).

${ }^{8}$ Aquí se aborda el modelo FED aplicando la metodología de Tom Copeland únicamente para ver las adaptaciones que se han hecho a este modelo consultar: Desmet (2000), Nygard (1999), Luehrman (1997).
} 
María Luisa Saavedra

Tabla 1

Valor intrínseco de la empresa

Valor presente de los flujos de efectivo disponibles

Menos: Valor presente de la deuda con costo

Fuente: Valuation. (Copeland, et al. 1994: 132).

Para implementar el modelo de flujo de efectivo disponible para la valuación de empresas $^{9}$ es necesario llevarlo a cabo en las siguientes etapas:

- Determinar el flujo de efectivo disponible

- Calcular la tasa de descuento apropiada

- Calcular el valor continuo ${ }^{10}$

Respecto de la determinación del flujo de efectivo algunos analistas usan el EBITDA como sustituto del mismo. Sin embargo, King (2002: 21) advierte acerca de las serias deficiencias que esto implica:

- El interés y los impuestos representan una prioridad que debe ser pagada virtualmente antes que ninguna otra cosa. Por lo tanto, EBITDA no es una medición de flujos de caja discrecionales.

- EBITDA suma otra vez el gasto de depreciación y amortización de activos previamente capitalizados. La actual aplicación contable indica que es un cargo no hecho en efectivo. Pero, ¿cuántas compañías pueden seguir adelante sin efectuar nuevos desembolsos de capital? EBITDA no considera esta situación.

- EBITDA desestima completamente los requerimientos de caja por el crecimiento de cuentas por cobrar e inventarios. Si una compañía está creciendo necesitará más inversión en estos rubros.

\footnotetext{
${ }^{9}$ Como se ha mencionado anteriormente aquí se usa el modelo de Copeland únicamente; sin embargo, si el lector quisiera consultar otras metodologías se recomienda ver Fernández (2003); este autor realiza un estudio comparativo de diez métodos diferentes para valuación de empresas, basados en flujo de efectivo descontado. Estos métodos son: Flujo de efectivo descontado al rendimiento requerido por el capital, Flujo de efectivo descontado al costo de capital, Flujo de efectivo descontado al costo de capital antes de impuestos, Valor presente neto ajustado, Ingreso residual descontado al rendimiento de capital, Valor económico agregado descontado al costo de capital, Flujo de efectivo ajustado al riesgo del negocio y al rendimiento requerido por los activos, Flujo de efectivo ajustado al riesgo del capital y al rendimiento requerido por los activos, el flujo de efectivo descontado a la tasa libre de riesgo y el flujo de efectivo descontado a la tasa libre de riesgo ajustado al capital. Después de realizar la aplicación empírica de todos los métodos concluye que todos los métodos arrojan el mismo valor utilizando los mismos supuestos.

${ }^{10}$ Esto bajo el supuesto que la empresa es un negocio en marcha y seguirá generando flujos indefinidamente.
} 
La valuación de empresas cotizadas en México, mediante la metodología del modelo de Flujo de Efectivo...

\subsection{Aplicación del Modelo de FED}

\subsubsection{En otros países ${ }^{11}$}

De acuerdo con estudios realizados por Heer y Koller (2000: 1) la determinación del valor de compañías cíclicas ${ }^{12}$-líneas aéreas, químicas, papel y acero, por ejemplo- pueden tener deficiencias en su valuación cuando se usa el modelo de Flujo de Efectivo Disponible. Sin embargo, un enfoque basado en probabilidades puede ayudar a alcanzar una estimación razonable. Para comprobar lo anterior realizaron un análisis a 36 compañías norteamericanas, cíclicas entre 1985 y 1997. Se encontró que el pronóstico no predice las utilidades de todo el ciclo dado, así como que el pronóstico no reconoce la existencia de un ciclo.

Se llega a la conclusión que, en esta situación es razonable construir un procedimiento de valuación de dos escenarios y ponderar sus valores. Bajo el primer escenario se asume con un $50 \%$ de probabilidad que el ciclo del pasado se repetirá y que la industria seguirá el mismo patrón en los próximos años. Bajo el segundo escenario se asume, también con un $50 \%$ de probabilidad, que la industria rompe su ciclo y sigue una nueva tendencia de largo plazo basada en el mejoramiento de su desempeño. El promedio ponderado de estos dos valores es el valor de la compañía.

Esta propuesta de valuación es bastante compleja, pero puede ayudar a llevar a una valuación más real cuando el negocio de la compañía es cíclico y en el cual es difícil tomar decisiones adecuadas. Esto nos da la pauta para suponer que se puede ser más efectivo en la valuación, usando escenarios macroeconómicos con probabilidades como lo hacen las grandes consultorías al proyectar las tendencias en los negocios.

\subsubsection{En México ${ }^{13}$}

Simón (1994: 245) realizó la evaluación del valor presente del flujo de efectivo de AHMSA, es decir, se aplicó el modelo de FED.

Los resultados arrojaron un valor presente neto de 81 mdd., con una tasa interna de retorno de $26 \%$, llegando a la conclusión que el proyecto permitirá recuperar la in-

\footnotetext{
${ }^{11}$ Para consultar más aplicaciones de FED en el extranjero ver: James y Koller (2000).

${ }^{12}$ Son aquéllas que no tienen ingresos estables en un periodo determinado, sino que tienen periodos de bajos ingresos y periodos de ingresos altos.

${ }^{13}$ Para consultar más aplicaciones en México véase Figueroa (1997), Jiménez (1997), Milla (1998).
} 
versión de 145 millones y tener un excedente de 81 mdd. Así también se observa que el proyecto es rentable dado que la tasa de retorno es mayor que el costo ponderado de capital.

Aunque los cálculos y las proyecciones para la aplicación de este modelo se apegaron a la realidad, no se consideró un elemento muy importante que es el valor continuo de los flujos de efectivo, el cual permite llegar a una valuación tomando en cuenta la vida indefinida de la empresa. Sin embargo, el resultado de la valuación demostró que no hubo subvaluación en la misma (tomando como referente el precio de venta de la empresa), lo cual implicaría que el cálculo del valor continuo no fue un elemento importante para este caso particular.

\subsection{Ventajas y desventajas del FED}

Ventajas:

- Contempla el potencial de crecimiento de la compañía. ${ }^{14}$

- Contempla las mejoras en eficiencia al considerar el rendimiento esperado.

- Refleja los rendimientos esperados basados en el riesgo del negocio o país.

- Permite realizar un análisis de sensibilidad completo.

- Al estar basado en flujos de efectivo, tasas de crecimiento y riesgo, específicos de la empresa, se evita la influencia de los errores de valuación del mercado (Damodaran, 1994: 16).

Desventajas:

- Se basa sólo en la habilidad de la compañía para generar flujos de efectivo, sin considerar la inversión total.

- Si la empresa no cotiza en bolsa será difícil estimar el riesgo (para estimar la tasa de descuento), dado que el modelo requiere que los parámetros de riesgo sean estimados del comportamiento histórico.

- La tasa del costo de capital refleja el riesgo de la empresa, de este modo los expertos eligen una tasa de descuento que corresponde al costo de capital de la empresa y no la tasa de rendimiento que requieren los inversionistas, induciendo así a errores de estimación (Nelson, 2000: 538).

\footnotetext{
${ }^{14}$ Entre quienes han intentado comprobar la validez del modelo tenemos a Christofi (1999) y Rappaport (1986). 
La valuación de empresas cotizadas en México, mediante la metodología del modelo de Flujo de Efectivo...

\section{Metodología para el cálculo del modelo de Flujo de Efectivo Disponible para determinar el valor de la empresa}

\subsection{Variables del modelo}

De acuerdo con la metodología aplicada por Tom Copeland et al. (1994: 135), las variables son:

a. Flujo de Efectivo. Es el excedente de efectivo operativo que genera la empresa.

b. Tasa de Crecimiento. Es la razón de crecimiento anual de la empresa; se toma generalmente el crecimiento en ventas.

c. Tasa de Descuento. Es la tasa del costo de capital promedio ponderado.

d. Valor Continuo. Es el valor de la empresa más allá del periodo explícito ${ }^{15}$ del pronóstico; se calcula como una perpetuidad. ${ }^{16}$ Se conoce como el valor de la empresa en marcha.

\subsection{Medición de las variables}

Específicamente para el cálculo del valor aplicando el Modelo FED a las empresas que cotizan en la Bolsa Mexicana de Valores, las variables se medirán así:

a. Flujo de efectivo $=$ Utilidad de operación + depreciación y amortización Impuestos

Esto en función de que Copeland et al. (1994: 95) señala que se debe de considerar el flujo de efectivo operativo, sin considerar gastos ni ingresos financieros, así también este flujo debe calcularse después de impuestos y depreciación.

b. Tasa de Crecimiento $=$ Se usará la tasa de crecimiento del sector medido con el

c. Tasa de Descuento $=$ Costo promedio ponderado de Capital.

El primer elemento del CCPP es el costo del capital propio; el modelo más usado para determinar este costo es el modelo de CAPM. Analizando la información histórica del Mercado de Valores Mexicano para el periodo 1991-2000, se observó que

\footnotetext{
${ }^{15}$ El periodo explícito del pronóstico es el periodo en el que proyectan los estados financieros de la empresa, con base en las perspectivas de crecimiento futuro.

${ }^{16}$ De acuerdo con Copeland, et al. (1995: 137), esta perpetuidad se calcula dividiendo el último flujo proyectado entre la diferencia de la tasa de rendimiento y la tasa de crecimiento.
} 
los rendimientos del mismo en algunos años de la serie histórica son negativos; esto hace que no sea posible usar el Modelo de CAPM para el cálculo del costo del capital propio.

Por lo anterior, se aplicará el método de la prima de riesgo, el cual de acuerdo, con Haime (1998: 152), consiste en aplicar una prima por riesgo al costo de la deuda de la empresa. Esta prima de riesgo se puede determinar por el diferencial que existe entre el rendimiento del mercado y la tasa libre de riesgo. Sin embargo, para México calcular así la prima de riesgo no es aplicable, ya que el mercado de valores es sumamente volátil y endeble, y así como puede tener rendimientos anualizados de más de $120 \%$ (año 1991), puede dar resultados negativos en los rendimientos anualizados (años 94, 98 y 2000). Por lo anterior, calcular de esa forma este diferencial nos puede llevar a resultados erróneos. Así, por ejemplo, tenemos que para el año 1999 el rendimiento del mercado fue $80.1 \%$, el rendimiento de $\operatorname{los} \operatorname{Cetes}^{17}$ a 28 días fue $21.41 \%$ y la prima de riesgo estaría del orden de $58.59 \%$, riesgo excesivo y fuera de la realidad.

Una alternativa de solución para la problemática planteada anteriormente, según Haime (1998: 153), es calcular la diferencia entre el promedio del rendimiento de las obligaciones de las empresas del ramo, contra el rendimiento de los Cetes. Sin embargo, para ser más específicos y evitar sesgos en esta investigación se esta planteando calcular el riesgo de cada empresa en particular. Para alcanzar este objetivo se hará el siguiente cálculo:

- Costo de deuda de la empresa - Tasa libre de riesgo ${ }^{18}=$ Prima de riesgo de la empresa El costo del capital propio, aplicando este método queda como sigue:

- Costo de Capital Propio = Costo de la Deuda de la Empresa + Prima de riesgo de la empresa

El segundo elemento para el cálculo del costo de capital promedio ponderado es el costo de la deuda de la empresa, el cual para efectos de esta investigación se determinará así:

$$
\text { Costo de la deuda }=\text { Intereses } / \text { Pasivo con costo }
$$

Este cálculo se hará así, en razón de que los únicos datos con que contamos son los que proporcionan los estados financieros publicados en la Bolsa Mexicana de Valores. Se decidió entonces usar la fórmula que muestra Weston (1994: 594) para el cálculo de la tasa anual efectiva simple:

\footnotetext{
${ }^{17}$ Certificados de la Tesorería de la Federación: Son instrumentos de deuda pública

${ }^{18}$ Para este efecto se considerará la tasa de Cetes anualizada a 28 días. 
La valuación de empresas cotizadas en México, mediante la metodología del modelo de Flujo de Efectivo...

$$
\text { Tasa anual efectiva } \text { simple }=\frac{\text { Interés }}{\text {------------ }} \text { Monto recibido }
$$

A este resultado se le hará el ajuste fiscal ${ }^{19}=$ Costo de la deuda $(1-\mathrm{T})$

Donde $\mathrm{T}$ es la tasa fiscal del Impuesto sobre la renta a la que está sujeta la empresa; en el caso mexicano $35 \%$.

Se ponderará el costo de cada componente de acuerdo a la proporción de los mismos dentro de la estructura de capital, multiplicando cada uno por su costo ya calculado, y se sumará estos productos para obtener el costo de capital promedio ponderado.

d. Valor Continuo $=$ Se calculará dividiendo el último flujo de efectivo entre la tasa de rendimiento menos la tasa de crecimiento.

Para calcular el flujo de efectivo disponible:

- Se proyectará el flujo de efectivo actual con el crecimiento anual del sector y se realizará la proyección por cinco años, dado que Figueroa (1997: 38) señala que el periodo de pronóstico explícito de los flujos de efectivo en los países emergentes debe ser corto; esto en razón de que son países con alta inestabilidad política y económica, en los cuales no es posible hacer pronósticos para el largo plazo.

- Se descontarán los flujos con la tasa del costo de capital promedio ponderado.

- Se calculará el valor de la empresa como la suma de los flujos disponibles, descontados a la tasa del costo de capital promedio ponderado.

Estos cálculos representados en una fórmula serían:

$$
\mathrm{VE}=\Sigma \frac{\mathrm{FE}}{(1+\mathrm{i})^{\mathrm{n}}}
$$
Donde:
$\mathrm{VE}=$ Valor de la empresa
$\mathrm{FE}=$ Flujos de efectivo disponibles
$\mathrm{i}=$ Costo de capital promedio ponderado

${ }^{19}$ Esto en razón de que los intereses son deducibles de impuestos. 
Es necesario señalar que no se considerará el cálculo del valor continuo, dado que Figueroa (1997: 35) recomienda para los países en desarrollo utilizar un enfoque conservador, de acuerdo con sus características de ser economías inestables y de alto riesgo.

\section{Determinación de la muestra}

La muestra está conformada por 71 empresas que cotizan en la Bolsa Mexicana de Valores, las mismas son aquéllas de las cuales se pudieron obtener los datos en las bases de datos de la propia BMV y de la Comisión Nacional Bancaria y de Valores para el caso de los Bancos.

Se consideró un periodo de estudio de 10 años (1991 a 2000) para reflejar el impacto de los cambios sexenales; los datos que se consideraron son anuales.

Se clasificaron las empresas de acuerdo a los sectores a los que pertenecen, según la clasificación que se usa en el medio bursátil.

Se decidió separar en 3 partes a las empresas del sector de la industria de la transformación, atendiendo a la similitud de su operación, tenemos entonces: Alimentos, Bebidas y Tabaco, Industria de la Transformación I, e Industria de la Transformación II.

Es necesario señalar que el total de empresas inscritas en la Bolsa Mexicana de Valores es de 170, pero que actualmente, según Yamashiro (2001: 20), sólo se encuentran operando en promedio 65 de ellas. Así también es importante saber que el IPC, ${ }^{20}$ al 31 de diciembre de 2000, se encontraba integrado por 34 empresas, ${ }^{21}$ las cuales se encuentran contenidas en esta muestra.

\section{Aplicación del modelo de Flujo de Efectivo Disponible a las empresas de la muestra}

Aplicando la metodología propuesta en el punto II, se determinó el valor de las empresas que conforman la muestra para cada uno de los 10 años objeto de estudio. Los datos de las variables financieras para cada empresa se tomaron de los Estados Financieros publicados en el anuario financiero de la Bolsa Mexicana de Valores.

Los valores promedio por sector aplicando el modelo FED se calcularon como un promedio simple del valor de las empresas que conforman cada sector; estos valores promedio se presentan en el anexo 1.

${ }^{20}$ Índice de Precios y Cotizaciones de la Bolsa Mexicana de Valores.

${ }^{21}$ Dato obtenido de: http:// www.bmv.com.mx, Bolsa Mexicana de Valores al 31 de diciembre de 2000 
La valuación de empresas cotizadas en México, mediante la metodología del modelo de Flujo de Efectivo...

Como podemos observar, en el anexo 1 (p. 109) tenemos los valores calculados para cada empresa y sector aplicando el modelo de FED para cada uno de los diez años del periodo objeto de estudio; así como el cálculo del valor promedio sectorial para cada año. Los espacios donde aparece n.d. significa que no se tuvo información disponible para realizar el cálculo del valor de estas empresas.

Tomando como patrón de referencia el precio de mercado ${ }^{22}$ de las empresas, se observa que por lo general el valor que arroja el modelo FED sigue una tendencia similar al precio de mercado. Las gráficas de la tendencia de los valores que arroja el modelo EVA y la tendencia del precio de mercado se puede ver en el anexo 2. (p. 108)

Como podemos ver, en las gráficas del anexo 2 existe una tendencia consistente en el modelo FED de mantener la misma tendencia que el precio de mercado; esto se debe a que este modelo considera, para la determinación del valor de la empresa, el flujo de efectivo que la misma genera determinado a partir de la utilidad de operación. La mayoría de las empresas que cotizan en la Bolsa Mexicana de Valores en el periodo de análisis, arrojaron un resultado de operación positivo, por esta razón los flujos también resultan positivos, lo que indica que existe una buena generación de recursos financieros a través del flujo de las empresas.

Al realizar un análisis de correlación de cada uno de los sectores entre los valores arrojados por el modelo FED y el precio del mercado se obtuvo el siguiente resultado:

Cuadro Núm. 1

\begin{tabular}{|l|c|}
\hline Sector & $\begin{array}{c}\text { Correlación FEDI } \\
\text { MERCADO }\end{array}$ \\
\cline { 1 - 1 } Alimentos, bebidas y tabaco & 0.8616 \\
Comercio & 0.8083 \\
\cline { 1 - 1 } Comunicaciones y transportes & 0.3689 \\
\cline { 1 - 1 } Construcción & 0.0879 \\
\cline { 1 - 1 } Controladoras & 0.6520 \\
Transf. I : Metal, minera, miner. no met. y siderúrgica & 0.8073 \\
\cline { 1 - 1 } Transf. II: Celulosa y papel y química & 0.8727 \\
\cline { 1 - 1 } Servicios Financieros & 0.4140 \\
\cline { 1 - 1 } Otros Servicios & 0.7529 \\
\hline
\end{tabular}

En el cuadro anterior podemos observar que gran parte de los sectores muestran una alta correlación con el modelo FED, lo cual estaría indicando que FED sería el modelo

${ }^{22}$ El precio de mercado se obtuvo del Anuario Bursátil de la Bolsa Mexicana de Valores. El mismo se conceptualiza como el precio de la acción multiplicada por el número de acciones en circulación. 
más adecuado para valuar estos sectores. Siendo únicamente el sector Construcción el que muestra una correlación poco significativa.

\section{Conclusiones}

El modelo de Flujo de Efectivo Disponible considera que el valor de la empresa en marcha estará dado por el flujo de efectivo libre disponible que se espera genere en el futuro, descontado a una tasa que refleje los riesgos del negocio. Las variables de este modelo son: Flujo de efectivo, excedente de efectivo operativo que genera la empresa; Tasa de crecimiento, razón anual de crecimiento de la empresa; Tasa de descuento, tasa del costo de capital promedio ponderado; Valor continuo, valor de la empresa más allá del periodo explícito del pronóstico.

Las principales ventajas de este modelo es que contempla el potencial de crecimiento de la compañía, así como las mejoras en eficiencia, por otro lado, al estar basado en flujos de efectivo, tasas de crecimiento y riesgo, específicos de las empresas, se evita la influencia de los errores de valuación del mercado. Las principales desventajas radican en que se basa sólo en la habilidad de la compañía para generar flujos de efectivo, sin considerar la inversión total, por lo que, si la empresa no cotiza en bolsa, será difícil estimar el riesgo.

Sin embargo se considera que el FED a pesar de las desventajas que presenta, es un modelo adecuado para valuar una empresa ya que sin flujo de efectivo éstas no serán capaces de financiar sus planes de expansión en un mercado competitivo.

De acuerdo con los resultados de esta investigación, el modelo FED es consistente en mantener un valor que sigue por lo general la misma tendencia que el precio de mercado. Esto se debe a que este modelo considera para la determinación del valor de la empresa el flujo de efectivo que la misma genera, el cual se determina a partir de la utilidad de operación. La mayoría de las empresas que cotizan en la Bolsa Mexicana de Valores en el periodo de análisis, arrojaron un resultado de operación positivo, por está razón los flujos también resultan positivos, esto indica que existe una buena generación de recursos financieros a través del flujo de las empresas.

En otras investigaciones en las que se ha aplicado este modelo, ${ }^{23}$ se ha demostrado que arroja valuaciones muy cercanas a los precios mercado de las acciones. Sin que

\footnotetext{
${ }^{23}$ James y Koller (2000: 80), realizaron una investigación para determinar el valor de las empresas aplicando el modelo FED a empresas Brasileñas. 
La valuación de empresas cotizadas en México, mediante la metodología del modelo de Flujo de Efectivo...

éste sea el caso de la presente investigación se puede considerar su resultado como coincidente en el sentido que es el valor que más se acerca al precio de mercado. De igual forma, al comparar nuestros resultados con los resultados arrojados para las mismas empresas en el mismo periodo, pero con el modelo de Valor Económico Agregado (Saavedra 2004: 39), se encontró que existe una tendencia consistente en dicho modelo de arrojar valores menores y, por lo general, negativos que el precio de mercado; lo cual se interpretó como una subvaluación. Así también aplicando el modelo de Black y Scholes (Saavedra 2005: 81) se observó una tendencia en él de arrojar valores mayores y positivos con respecto al precio de mercado; lo cual se interpretó como una sobrevaluación.

La aplicación de este modelo al conjunto de empresas mexicanas por el periodo bajo estudio permite definir que los valores intrínsecos que arrojan las empresas con este modelo siguen la tendencia del mercado, lo cual podría indicar que es un modelo adecuado para determinar el valor en este tipo de empresas.

\section{Bilbiografía y Hemerografía}

Bolsa Mexicana de Valores (2001). Anuario Bursátil de la Bolsa Mexicana de Valores. México: Bolsa Mexicana de Valores, S.A. de C.V.

Bolsa Mexicana de Valores (2001). Anuario Financiero de la Bolsa Mexicana de Valores. México: Bolsa Mexicana de Valores, S.A. de C.V.

Carvallo, Roberto (1995). Valuación de Empresas. Tesina para obtener el título de Licenciado en Administración. México: ITAM.

Copeland, Tom, Tim Koller y Murrin Jack (1994). Valuation: Measuring and managing the value of companies. EUA: McKinsey and Company.

Copeland, Thomas y Weston Fred (1995). Finanzas en Administración. México: Mc Graw Hill.

Copeland, Tom (2000). Valuation: Measuring y Managing the Value of Companies $\left(3^{\text {rd }}\right.$ ed). EUA: Mc. Kinsey \& Company, Inc. John Wiley \& Sons, Inc.

Christofi, Andreas, Petros Christofi, Donald Moliver y Lori Marcus (1999). Evaluating common stocks using value lines projected cash flow and implied growth rate. Journal of Investing, Spring, 1, pp. 38-45.

Damodaran, Aswath (1994). Damodaran on Valuation. Security Analysis for Investment and Corporate Finance. EUA: John Wiley \& Sons, Inc. 
Desmet, Driek (2000). "Valuing Dot-coms", The McKinsey Q., EUA. núm. 1. pp. 148-157.

Fernández, Pablo (2003). Equivalence of Ten Methods for Valuing Companies by Cash flows Discounted. Barcelona: IESE Business School, Universidad de Navarra.

FigueroA, José (1997).Valuación de Empresas para Países en Desarrollo. México: UNAM, Facultad de Ingeniería. Tesis de Maestría en Ingeniería (Planeación).

Franks, Julian, J. Broyles y W. Carleton (1985). Corporate Finance. EUA: Kent Publishing Company.

Haime, Luis (1998). Reestructuración integral de empresas. México: Isef.

Heer, Marco y Timothy Koller (2000). Valuating Cyclical Companies. The Mc Kinsey Quarterly, núm. 2. pp. 78-85, EUA.

James, Mimi y Timothy Koller (2000). Valuation in Emerging Markets, The McKinsey Quartely, núm. 4. p. 80. EUA.

JiMÉnEZ, José (1997). Estudio de un caso de valuación de empresas. México: UNAM, Facultad de Ingeniería,Tesis de grado de Maestría en Ingeniería (Investigación de operaciones).

King, Alfred (2002). Advertencia: el uso del EBITDA puede ser peligroso para su carrera. Veritas, Febrero. México. pp. 20-22.

Luehrman, Timothy (1997). Using APV: A Better Tool for Valuating Operations. Harvard Business Review, EUA. Mayo-junio pp. 145-154.

Mascareñas, Pérez-IÑigo (2000). Fusiones y Adquisiciones de empresas (3ra ed.). España: Mc Graw Hill.

Milla, Sindy (1998). El Análisis Fundamental y la Valuación de Acciones en México. México: UNAM, Facultad de Contaduría y Administración, Tesis de Grado de Maestría en Finanzas.

Nelson, James (2000). Opposing discounted cash flow analysis, Defense Counsel Journal, Chicago. Vol. 67, issue: 4 pp. 536-540.

Nygard, Wayne y Christopher Razaire (1999). Probability-based DCF: An alternative to point-value estimates. EUA: The Appraisal Journal Chicago, vol. 67, issue: 1 (pp. 68-74).

Opiela, NANCY (2000). What you need to know about valuing your practice. Denver: Journal of Financial Planning; Vol. 13, issue: 2, febrero, pp. 60-67. 
La valuación de empresas cotizadas en México, mediante la metodología del modelo de Flujo de Efectivo...

Penman, Stephen (1998). A Comparison of Dividend, Cash Flow, and Earnings Approaches to Equity Valuation. EUA: Contemporary Accounting Research, Vol. 15, núm. 3, p. 343-383.

RAPPAPORT, Alfred (1986). Creating Shareholder value. Nueva York: The Free Press.

SaAvedra García, María Luisa (2004). La Valuación de empresas en México. Aplicación del Modelo de Valor Económico Agregado 1991-2000. En Contaduría y Administración, núm. 214, septiembre-diciembre pp. 25-50.

SaAVedra García, María Luisa (2005). Aplicación empírica del modelo de Black y Scholes en México: 1991-2000. Revista Contaduría y Administración, núm. 217 , septiembre-diciembre pp. 65-95.

SALAS, HÉctor (2002). Integración e Inestabilidad Financiera. Capítulo: Valuación de Empresas. En A. Girón y E. Correa (Coordinador), México: UNAM-IIEc, pp. 23-32.

Simón, Nadima (1994). Evaluación Organizacional: El Caso de Altos Hornos de México, S.A. después de la privatización. México: UNAM-FCA, Tesis de Grado de Doctorado en Administración.

Taggart, James, Peter Kontes y Michael Mankins (1994). The Value Imperative. Managing for Superior Shareholder Returns. Nueva York: The Free Press.

Weston, Fred y Eugene Brigham (1994). Administración Financiera. México: McGraw Hill.

Thierauf, Robert y Richard Grosse (1987). Toma de decisiones por medio de Investigación de Operaciones. México: Limusa.

Van Horne, James (1993). Administración Financiera. México: Prentice Hall.

Yamashiro, Celina (2001, junio 25). Las Empresas en la Bolsa Mexicana de Valores. El Financiero, Sección de Negocios p. 20. 
Anexo 2
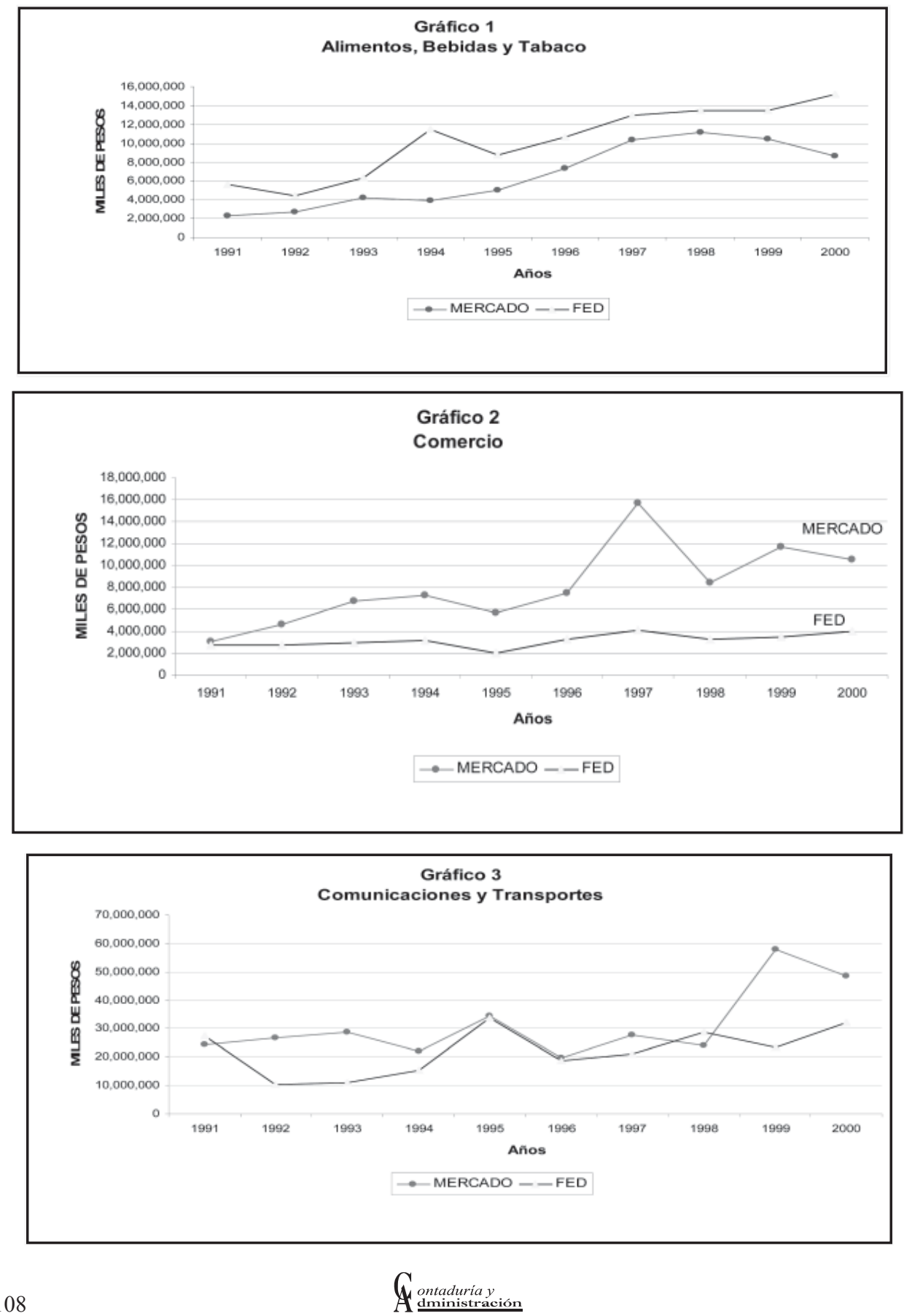


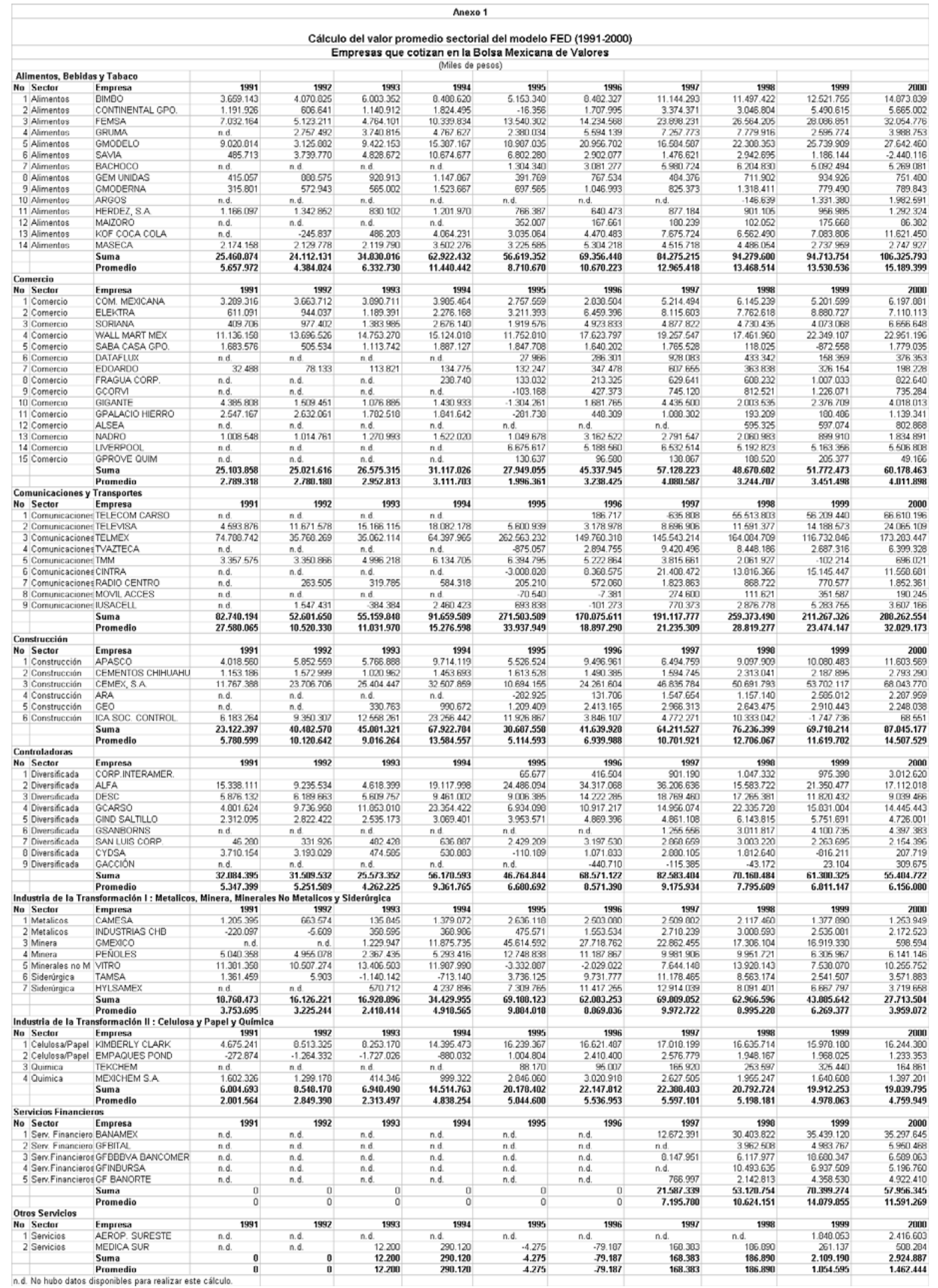


La valuación de empresas cotizadas en México, mediante la metodología del modelo de Flujo de Efectivo...
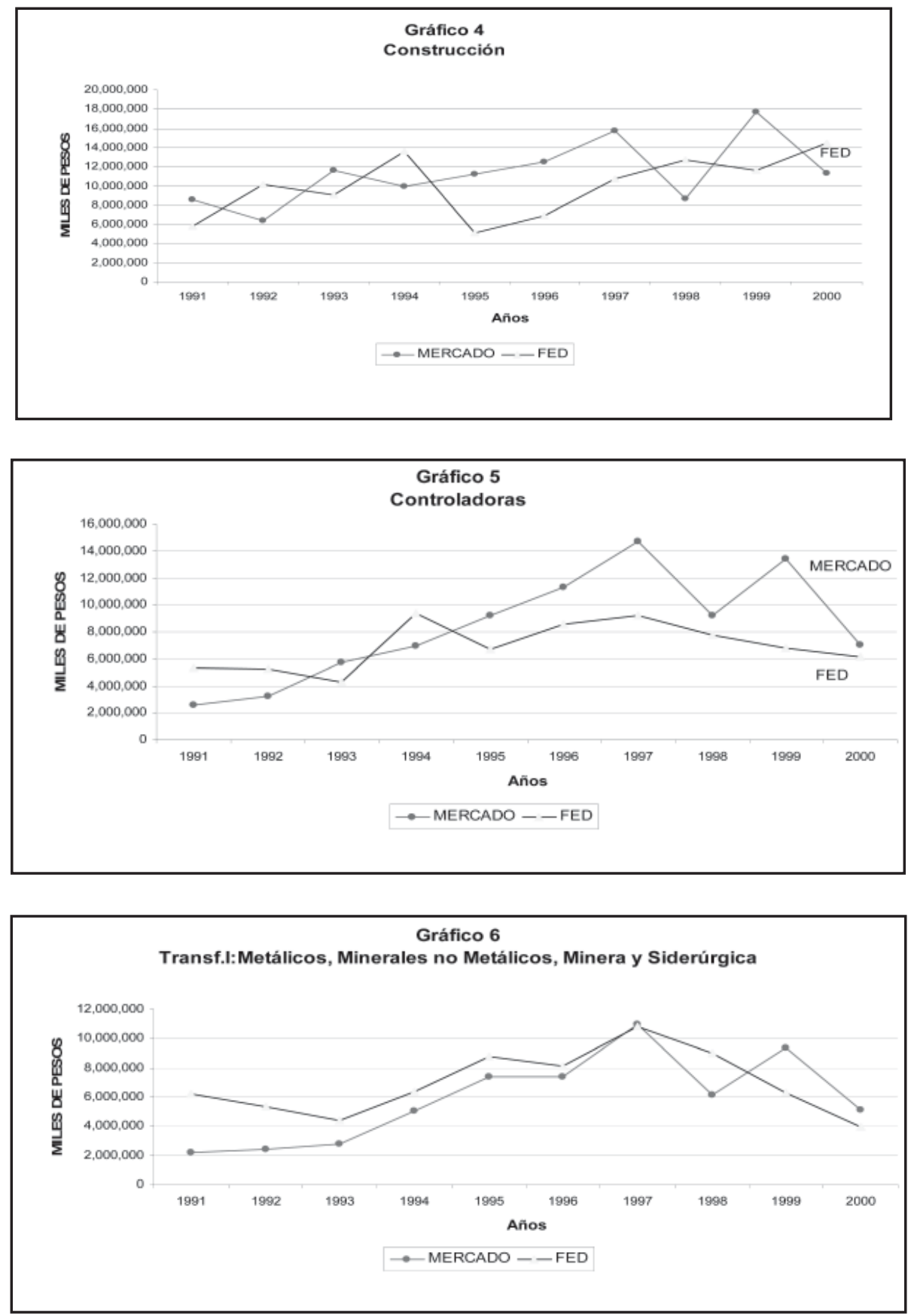

Núm. 223, septiembre-diciembre 2007: 91-112 
María Luisa Saavedra
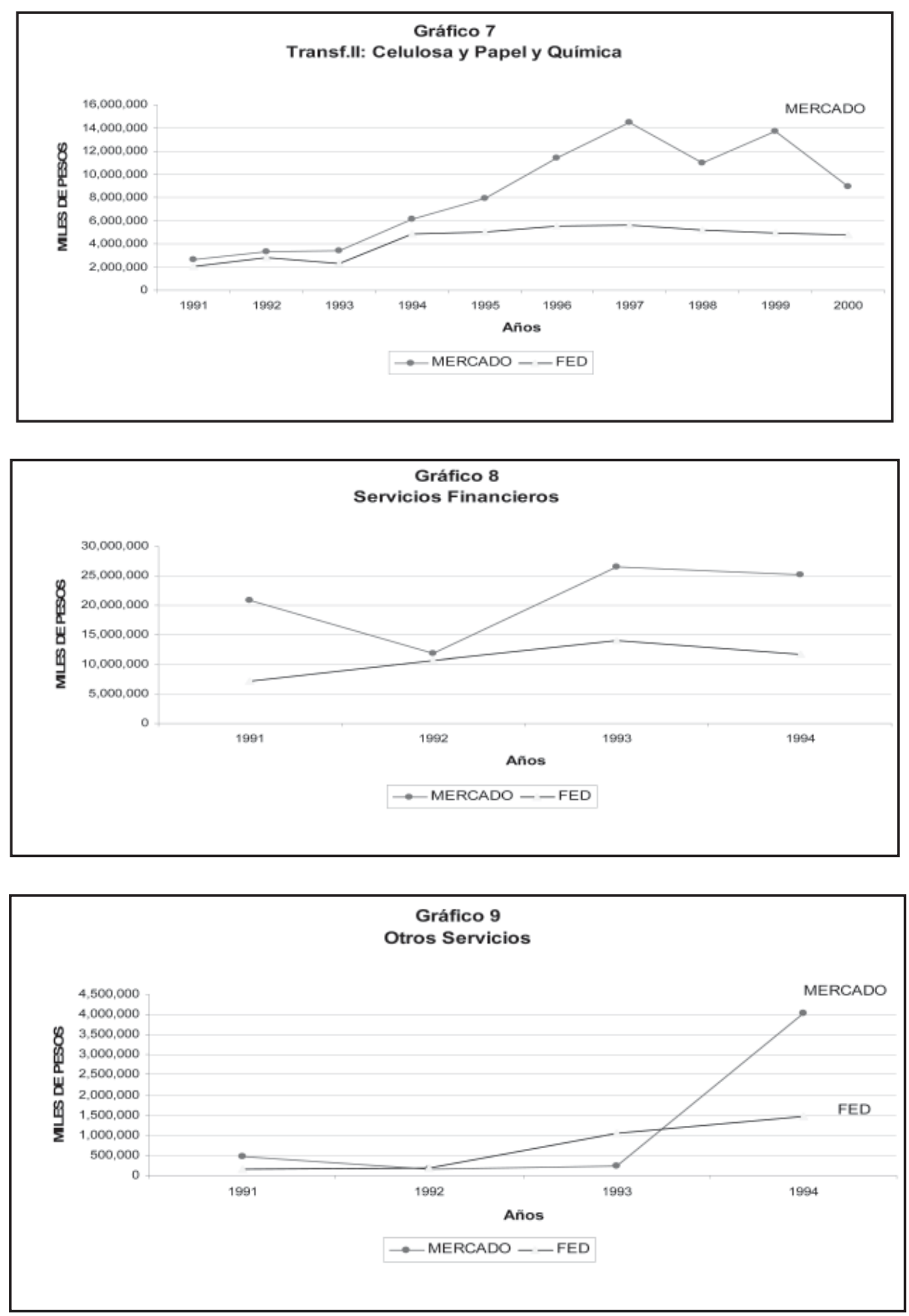

112

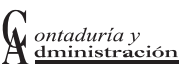

